\title{
Una visión actualizada sobre la síntesis, escalado y aplicaciones de las nanoemulsiones dobles ${ }^{1}$
}

\section{An updated overview about the synthesis, scaling-up and applications of double nanoemulsions}

\author{
F. A. Silva y P. A. Oyarzún \\ Recibido: enero 21 de 2021 - Aceptado: noviembre 15 de 2021
}

\begin{abstract}
Resumen - Las nanoemulsiones son sistemas emulsionados de tamaño nanométrico. Estas pueden ser producidas mediante diversos métodos, los cuales se pueden clasificar como métodos de baja y alta energía. La disminución en el tamaño de glóbulo formado permite que estas puedan penetrar con mayor facilidad diferentes barreras como la piel o la barrera gastrointestinal. De este modo la encapsulación de compuestos activos a través de nanoemulsiones permite mejorar su biodisponibilidad. Además de ofrecer protección frente a factores ambientales como temperatura, humedad o radiación UV. Asimismo, es posible formular nanoemulsiones dobles con el fin de encapsular moléculas de naturaleza hidrofílica y lipofílica en un solo glóbulo. Este proceso de formación requiere un análisis integral que incluye aproximaciones físicas y termodinámicas.
\end{abstract}

Palabras clave- Nanoemulsión, Nanoemulsión doble, Microemulsión, emulsificación, emulsión sub-micrométrica

Abstract - Nanoemulsions are nano-sized emulsified systems. They can be produced by different methods, which can be classified as low and high energy methods. The decrease in the size of formed globules allows them to penetrate different barriers such as the skin or the gastrointestinal barrier. Thus, the encapsulation of active compounds through nanoemulsions makes it possible to improve their bioavailability and to offer

${ }^{1}$ Producto derivado del proyecto de investigación FONDEF VIU18E0030, apoyado por la Universidad San Sebastián sede Tres Pascualas, Concepción y el laboratorio de Nanobiotecnología y optoelectrónica (USS).

F. A. Silva, Facultad de Ingeniería y Tecnología, Universidad San Sebastián, Sede Concepción, Chile, email: fsilvaj1991@gmail.com

P. A. Oyarzún, Facultad de Ingeniería y Tecnología, Universidad San Sebastián, Sede Concepción, Chile, email: patricio.oyarzun@uss.cl

Como citar este artículo: Silva, F. A., Oyarzún, P. A., Una visión actualizada sobre la síntesis, escalado y aplicaciones de las nanoemulsiones dobles, Entre Ciencia e Ingeniería, vol. 15, no. 30, pp. 30-40, julio-diciembre 2021. DOI: https://doi.org/10.31908/19098367.2095.

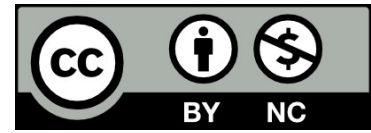

Attribution-NonCommercial 4.0 Intenational (CC By-NC 4.0) protection against environmental conditions such as temperature, humidity, or UV radiation. In the same way, it is possible to formulate double nanoemulsions to encapsulate hydrophilic and lipophilic molecules in one single globule. Their formulation process requires a comprehensive analysis involving physical and thermodynamic approaches.

Keywords- Nanoemulsion, Double Nanoemulsion, Microemulsion, emulsification, sub-micrometric emulsion

\section{INTRODUCCIÓN}

T as emulsiones son sistemas coloidales que permiten la Uunión o dispersión de fases inmiscibles entre sí. Esto se logra gracias al uso de estabilizantes, los cuales disminuyen la tensión superficial entre las fases y forma una capa entre la fase dispersa y la fase continua. Estos tipos de coloide son utilizados en diversas industrias como la cosmética, farmacéutica, alimentos, agricultura, entre otras [1]. En general el tamaño de las emulsiones es micrométrico y pueden ser observadas mediante microscopía óptica. Existen algunas estrategias para reducir el tamaño de las emulsiones, los cuales van desde invertir fases hasta incrementar la energía para romper los glóbulos formados. Estas reciben el nombre de nanoemulsiones y microemulsiones según el método de formación. En particular las nanoemulsiones son emulsiones nanométricas para las cuales algunos autores incluso han definido un diámetro máximo $<1000 \mathrm{~nm}$. Las nanoemulsiones permiten encapsular diversos compuestos activos entregando ciertas ventajas con respecto a las emulsiones. Debido a la disminución en el tamaño de glóbulo son capaces de aumentar el área de contacto, aumentar la disponibilidad de los compuestos activos, protección contra factores ambientales y liberación controlada de estos [2]. Las nanoemulsiones son flexibles y permiten la incorporación de diversas fases, siendo posible formular nanoemulsiones dobles del tipo W/O/W y $\mathrm{O} / \mathrm{W} / \mathrm{O}$, las mayormente descrita. Estas facilitan la unión de compuestos activos lipofílicos e hidrofílicos en un solo glóbulo [3]. La existencia de la doble interfase requiere un análisis comprehensivo para su formulación y se deben tomar 
en cuenta consideraciones fisicoquímicas para lograr estabilizar los glóbulos formados. A continuación, se describen aspectos básicos para la formación de nanoemulsiones simples y conceptos claves para la formulación de nanoemulsiones dobles y sus aplicaciones.

\section{ANÁLISIS GLOBAL DE MICROEMULSIONES, NANOEMULSIONES Y NANOEMULSIONES DOBLES}

Los términos nanoemulsiones y microemulsiones pueden ser confundidos por el prefijo de cada uno de estos. El prefijo nano- hace referencia al tamaño nanométrico del glóbulo formado $\left(10^{-9} \mathrm{~m}\right)$, mientras que micro- lo hace a su correspondiente prefijo $\left(10^{-6} \mathrm{~m}\right)$. Sin embargo, la diferencia no recae en lo anteriormente descrito. Más bien, se deben considerar otros elementos para la clasificación de uno u otro grupo [4]. Si bien, para nanoemulsiones producidas de manera convencional por métodos de alta energía es posible simplificar el análisis mediante generalizaciones de las ecuaciones 1 y 2 . En el caso de nanoemulsiones y microemulsiones fabricadas a través métodos de baja energía se requiere un análisis más fino, debido a que ambos tipos de emulsiones descritas están regidas por un mecanismo de formación universal [5]. Anton \& Vandamme [6] realizaron una clara diferenciación entre los términos, describiendo la formación de nanoemulsiones mediante dos mecanismos (I) Métodos de alta energía como homogenización de alta velocidad, ultrasonido u homogenización de alta presión. La alta energía suministrada a la emulsión provoca la disminución del tamaño de glóbulo formado e incrementa el área interfacial entre la fase dispersa y continua. (II) Método de baja energía emulsificación espontanea. Este método consiste en dispersar el o los surfactantes en la fase contraria a la que son afines. En el caso de las nanoemulsiones O/W el surfactante hidrofílico se añade en la fase oleosa. Esta es calentada hasta $\operatorname{los} 30^{\circ} \mathrm{C}$, mientras que la fase dispersa se mantiene a temperatura ambiente. Cuando ambas fases se unen, se genera rápidamente un glóbulo de tamaño nanométrico.

Algunos estudios en donde se utilizan estos métodos se muestran en la Tabla I.
Termodinámicamente, las nanoemulsiones son coloides inestables que no se generan espontáneamente. Sin embargo, debido al mecanismo de formación universal, mencionado anteriormente, es posible obtener nanoemulsiones mediante métodos de baja energía. Un análisis termodinámico general permite explicar la formación de nanoemulsiones a través de métodos de alta energía. Según lo presentado por McClements [11], Hunter [12], y Tadros [13], la emulsificación y nanoemulsificación se ve favorecida por dos componentes: energía libre de interfase y entropía de configuración, Ec. (1) y Ec. (2), respectivamente.

$\Delta G=H-T \Delta S$

$\Delta G_{\text {Formación }}=\Delta G_{\text {Interfase }}-T \Delta S_{\text {configuración }}$

Donde el término $\Delta \mathrm{G}_{\text {Interfase }}$ representa la energía libre de la interfase o trabajo requerido para incrementar el área de la interfase. Esta última resulta de la consideración de que no existen cambios de volumen e intercambio de calor en el sistema y el término asociado a la energía libre es igual a la energía interna. Láng [14] dentro del marco del modelo de Gibbs define la tensión superficial según la Ec. (3).

$$
\gamma=\left(\frac{\partial U^{E}}{\partial A}\right)_{S^{E}, n_{1}^{E} \ldots, n_{m}^{E}}
$$

Donde $\mathrm{U}^{\mathrm{E}}$ es energía interna en exceso, $\mathrm{A}$ es área superficial, $\mathrm{S}^{\mathrm{E}}$ es entropía en exceso, y $n_{m}$ es el componente $m$ en exceso. Sin embargo, Láng [14] se refiere a la Ec. (3) como poco práctica en términos experimentales debido a las variables presentes en ella. Tras una serie de transformaciones es más conveniente expresar la tensión superficial en términos de energía libre de Gibbs o Helmholtz, Ec. (4).

$$
\gamma=\left(\frac{\partial G}{\partial A}\right)_{T, p, n_{1} \ldots n_{m m}}=\left(\frac{\partial F}{\partial A}\right)_{T, V, n_{1} \ldots n_{m}}
$$

De manera simplificada la $\Delta \mathrm{G}_{\text {Interfase }}$ puede ser representada mediante la Ec. (5).

$$
\Delta G_{\text {Inter fase }}=\gamma \Delta A
$$

\begin{tabular}{|c|c|c|c|}
\hline Nanoemulsión & Método de emulsificación & Descripción & Referencia \\
\hline $\mathrm{O} / \mathrm{W}$ & $\begin{array}{c}\mathrm{HSH} \\
(\mathrm{HPH}), \mathrm{HSH}+\mathrm{HPH}\end{array}$ & $\begin{array}{l}10 \% \mathrm{w} / \mathrm{w} \text { DHA } \\
\text { sobre solución acuosa de Tween } 40 \text {. }\end{array}$ & {$[7]$} \\
\hline $\mathrm{O} / \mathrm{W}$ & $\begin{array}{l}\text { Emulsión gruesa HSH } \\
\text { Nanoemulsión HPH }\end{array}$ & $\begin{array}{c}3.75 \% \mathrm{w} / \mathrm{w} \text { Span } 80 \text { en aceite de soya } \\
1.25 \% \text { w/w Tween } 80(1: 9) .\end{array}$ & [8] \\
\hline $\mathrm{O} / \mathrm{W}$ & Emulsificación espontánea & $\begin{array}{c}\text { Adición de Labrafil M1944 Cs y Cremophor ELP } \\
\text { sobre la fase oleosa. }\end{array}$ & {$[5]$} \\
\hline $\mathrm{O} / \mathrm{W}$ & Emulsificación espontánea & $\begin{array}{c}\text { Adición de Kolliphor HS15 y Span } 80 \text { sobre la fase } \\
\text { oleosa. }\end{array}$ & [9] \\
\hline $\mathrm{O} / \mathrm{W}$ & $\begin{array}{c}\text { Emulsión gruesa HSH } \\
\text { Nanoemulsificación por ultrasonido }\end{array}$ & $\begin{array}{c}\text { Adición de Tween } 80 \text { y lecitina de soya en la fase } \\
\text { continua. }\end{array}$ & {$[10]$} \\
\hline
\end{tabular}

TABLA I

ESTUDIOS REFERENTES A LA FORMACIÓN DE NANOEMULSIONES

${ }^{*} H S H=$ High Speed Homogenizer, HPH=High Preassure Homogenizer 
La tensión superficial $\gamma$ tiene unidades de $\mathrm{N} / \mathrm{m}$ o $\mathrm{mN} / \mathrm{m}$ y $\Delta \mathrm{A}$ es la diferencia entre el área de interacción de los componentes no emulsionados $\left(\mathrm{A}_{1}\right)$ y la emulsión/nanoemulsión $\left(\mathrm{A}_{2}\right)$, donde $\mathrm{A}_{2}>>\mathrm{A}_{1}$.

Por otra parte, la entropía de configuración hace referencia a la configuración o curvatura que adquiere el surfactante utilizado. En emulsiones y nanoemulsiones, el estabilizante se curva generando un "radio de curvatura". Existen dos ecuaciones útiles para entender el efecto de lo anterior sobre la ecuación general, la primera es la ecuación de presión de Laplace (Ec. 6.), la otra es la ecuación de "entropía de configuración" (Ec. 7).

$\Delta p=\gamma\left(\frac{1}{r_{1}}+\frac{1}{r_{2}}\right)=\gamma\left(\frac{2}{r}\right)$

$\Delta S_{\text {Configuración }}=\frac{n k}{\phi}(\phi * \ln \phi+(1-\phi) * \ln (1-\phi))$

Donde $\Delta p$ es la diferencia de presión interior y exterior (medida en Pa), $r$ es el radio del glóbulo formado, $n$ es el número de glóbulos en la dispersión coloidal y $\Phi$ es la fracción de volumen de la fase dispersa. En términos generales el proceso de nanoemulsificación será viable si la energía libre total es negativa. Por lo tanto, la entropía de configuración debe ser $\Delta \mathrm{S}_{\text {configuración }}>0$ y la energía de interfase debe ser minimizada mediante la introducción de surfactantes que disminuyen la tensión superficial y la presión de Laplace. Sin embargo, a medida que disminuye el tamaño de glóbulo la presión de Laplace aumenta, esta presión actuará como resistencia a cualquier efecto externo, requiriendo una mayor cantidad de energía para disminuir el tamaño de glóbulo [15]. Adicionalmente, existen diversos factores que determinan una configuración favorable del punto de vista energético como la temperatura, sinergia entre surfactante/surfactante o surfactante/co-surfactante, la distribución y longitud de la porción hidrofílica/lipofílica de los surfactantes, y la interacción de los tensioactivos con la fase continua [16].

En contraposición con las nanoemulsiones, las microemulsiones son sistemas coloidales termodinámicamente estables. La baja energía necesaria para lograr la formación de microemulsiones las hacen ser una interesante alternativa en comparación con los métodos de alta energía descritos para nanoemulsiones. La unión Internacional de Química Pura y Aplicada (IUPAC) define a las microemulsiones como "Una dispersión hecha de agua, aceite y surfactante(s), que es isotrópica y termodinámicamente estable con dominio del diámetro de la dispersión variando aproximadamente desde 1 a $100 \mathrm{~nm}$, usualmente de 10 a $50 \mathrm{~nm}$ " [17]. Estas se forman exclusivamente por métodos de baja energía los cuales incluyen la emulsificación espontánea y métodos de inversión por temperatura (PIT) e inversión de fases (PIC), para los cuales Solans \& Solè [18] refieren como principal diferencia la difusión rápida de los surfactantes entre las diferentes fases y cambios espontáneos en la curvatura de estos, respectivamente. Los métodos de inversión de fases pueden ser utilizados tanto para la formación de nanoemulsiones como microemulsiones. Asimismo, es posible formar una nanoemulsión a partir de la síntesis primaria de una microemulsión que posteriormente es diluida [18]. Esta es generada tras alcanzar un nuevo equilibrio que provoca la salida de moléculas de surfactante desde la interfase y un aumento en el tamaño de glóbulo. Esto ha sido una de las bases para diferenciar una microemulsión de una nanoemulsión, al asumir que esta última requiere una menor cantidad de tensioactivo. Sin embargo, de manera crítica, una mayor o menor cantidad de surfactante solo abarca una región diferente en el diagrama de fases ternario, en el cual también se encuentran otras estructuras. Por esto, en esencia una nanoemulsión preparada mediante métodos de baja energía es una microemulsión que puede ser obtenida al analizar un diagrama de fases ternario.

Anton \& Vandamme [5] realizaron una comparación entre el método PIT y el de emulsificación espontanea, evidenciando que solo difieren en el factor razón surfactante/aceite $(\mathrm{SOR}=$ surfactante/oil ratio), proponiendo que el método PIT se rige por el mismo mecanismo de emulsificación espontanea tras alcanzar la temperatura crítica. A partir de lo anterior, está ampliamente aceptado que las microemulsiones son formuladas con concentraciones altas de surfactantes $(\sim 20 \%)$, por el contrario, las nanoemulsiones requieren una menor cantidad de surfactantes ( 10) [19]-[20]. En particular, se ha descrito que las microemulsiones no solo adoptan una configuración globular la cual recibe el nombre de swollen micelles. Más bien, es posible encontrar estructuras laminares, bicontinuas, hexagonales, cilíndricas o líquidos cristalinos. Dichas estructuras se presentan para ciertas condiciones que pueden ser exploradas mediante el diagrama de fases ternario (aceite, agua, surfactante/surfactante o surfactante/co-surfactante). Justamente, es en este diagrama en donde se sitúan las regiones Winsor (I-IV) que representan la formación de microemulsiones en equilibrio, exceso de fase continua o estructura bicontinua [6]. Alternativamente a la exploración del sistema de fases ternario, es posible predecir el tamaño y viscosidad de microemulsiones mediante el método descrito por Acosta et al. [21] y Kiran \& Acosta [22]. Aproximaciones más modernas incluyen el modelado in silico de microemulsiones basado en la teoría termodinámica y minimización de la energía libre de Gibbs [23] y la simulación mediante dinámica molecular combinada con diversos algoritmos permiten calcular parámetros como potenciales químicos, coeficientes de actividad, densidad de carga de la superficie y coeficientes de partición de los componentes de la microemulsión y la fase continua [24]. El trasfondo termodinámico para la formación de microemulsiones puede ser analizado en [25], [26], [27], [28]-[29].

En el caso de las nanoemulsiones dobles es posible realizar formulaciones que incluyan estrategias de alta y baja energía, tal como lo demostraron Ding et al. [30]. Inicialmente los autores generaron una nanoemulsión tipo $\mathrm{W} / \mathrm{O}$ mediante homogenización rotor/estator y microfluidizador, logrando un tamaño de glóbulo de $50 \mathrm{~nm}$. Posteriormente se utilizó el método de emulsificación espontanea para obtener la nanoemulsión $\mathrm{W} / \mathrm{O} / \mathrm{W}$. 


\section{ASPECTOS FÍSICOS EN LA FORMACIÓN DE NANOEMULSIONES POR MÉTODOS DE ALTA ENERGÍA}

La formación de nanoemulsiones también puede ser explicada mediante ecuaciones físicas y números adimensionales. Existe una rica teoría para describir y predecir el tamaño de glóbulo en las emulsiones tradicionales frente a flujos laminares y turbulentos. Parámetros como viscosidad de la fase continua, viscosidad de la fase dispersa, tensión superficial, entre otros; son esenciales para entender el proceso [31]. En líneas generales, el proceso de emulsificación y la disminución en el tamaño de glóbulo está gobernado por dos números adimensionales. Estos son el número de Weber que es la razón entre las fuerzas inerciales y de tensión superficial (Ec. 8).

$W e=\frac{\tau_{\text {aplicado }} d}{\sigma}$

Donde $\tau$ es el esfuerzo de corte, $\mathrm{d}$ es el diámetro del glóbulo y $\sigma$ es la tensión superficial. $\mathrm{Y}$ el número de Ohnesorge que es la razón entre la viscosidad de la fase dispersa (fuerzas viscosas) y tensión superficial (Ec. 9).

$O h=\frac{\mu_{d}}{\sqrt{\rho_{d} \sigma d}}$

Donde $\mu_{\mathrm{d}}$ es la viscosidad de la fase dispersa y $\rho_{\mathrm{d}}$ es la densidad de la fase dispersa. Dichos números adimensionales se relacionan según la Ec. (10)

$W e_{\text {crit }}=C_{1}(1+f(O h))$

Gupta et al. [32] resumen y desarrollan el efecto de $f(\mathrm{Oh})$ sobre la Ec. (10). En los casos donde $f(O h) \ll 1$, se considera que las fuerzas viscosas son despreciables y $W e_{\text {crit }}$ se convierte en la constante $\mathrm{C}_{2}$ que es similar a la ecuación 6 y cuyo tamaño de glóbulo puede ser explicado por la Ec. (11). que surge de sustituir $\tau \sim \mu_{\mathrm{c}} \gamma$.

$d=\frac{c_{1} \sigma}{\mu_{r} \gamma}$

$\mu_{c}$ es la viscosidad de la fase continua, $\gamma$ es la velocidad de corte $\mathrm{y}_{1}$ o número de capilaridad es en función de la razón de viscosidad entre las fases. En este escenario la disminución en el tamaño de glóbulo se dará en regímenes de flujo laminar si las razones de viscosidad entre la fase dispersa y continua son bajas ( $<4$ para velocidad de corte normal), y si la escala de tiempo de la deformación es menor a la escala de tiempo del flujo [33]. Por el contrario, si $f(O h) \gg 1$, la viscosidad de la fase dispersa tiene efectos significativos sobre el tamaño de glóbulo y mayor estrés inercial es requerido para obtener glóbulos nanométricos. Este régimen es denominado viscosoturbulento y está gobernado por $\tau=\sqrt{\mu_{c} R_{c} \varepsilon}, \rho_{c}$ es la densidad de la fase continua y $\varepsilon$ es la densidad de potencia de entrada definida como la disipación de potencia por unidad de masa del fluido [34]. Luego la relación entre los números adimensionales Weber y Ohnesorge se da mediante la Ec. (12).

$W e_{\text {crit }}=C_{4} O h^{0.4}$

Donde $W e_{c r i t}$ es igual a $\frac{\sqrt{W_{\varepsilon} \rho_{c} a d}}{\sigma}$. Para la constante $\mathrm{C}_{4}$, Gupta et al. [32] hace referencia a la existencia de un colapso universal en el rompimiento del glóbulo que en el caso de homogenización por alta presión es 0.063 y homogenización por ultrasonido es 0.057 . No se menciona el valor de la constante para homogenización de alta velocidad o microfluidizador. Por este motivo, el cálculo de $\varepsilon$ es característico para cada técnica de homogenización. Sin embargo, debido al colapso universal, el valor de $\varepsilon$ debe ser de orden similar para las diferentes técnicas mencionadas. Finalmente, para regímenes de flujo turbulento y alta viscosidad de la fase dispersa como en nanoemulsiones tipo O/W, el tamaño de glóbulo está dado por la Ec. (13).

$d=\frac{\sigma^{\frac{5}{6}} \mu_{d}^{\frac{1}{3}}}{\left(\rho_{d} \sigma\right)^{1 / 5}\left(\mu_{c} \rho_{c}\right)^{5 / 12}}$

\section{SÍNTESIS DE NANOEMULSIONES DOBLES}

El método más utilizado para la síntesis de emulsiones y nanoemulsiones dobles es el método secuencial en donde se genera una nanoemulsión inicial la cual, posteriormente, es añadida en la fase dispersa final. Generalmente se ha descrito este método para la formación de nanoemulsiones $\mathrm{W} / \mathrm{O} / \mathrm{W}$ ó $\mathrm{O} / \mathrm{W} / \mathrm{O}$. Requiere el estudio y selección de emulsionantes que puedan estabilizar las tres fases. La preparación de la fase final $\mathrm{W}_{2}$, se realiza añadiendo un emulsionante hidrofílico que estabilice la nanoemulsión W1/O/W2 [35]-[36]. La selección de emulsionantes que permitan la síntesis de una primera fase W1/O altamente estable es crucial para la estabilización de la doble emulsión, este debe cumplir con propiedades como expansibilidad, compresibilidad y dar balance entre la presión osmótica y Laplace [35], especialmente para esta última que en nanoemulsiones incrementa a medida que disminuye el tamaño de glóbulo. Otras estrategias que se han diseñado para la formación de nanoemulsiones dobles es la mezcla entre métodos de alta y baja energía, la cual fue propuesta por Ding et al. [30].

Existen una serie de aproximaciones que se han propuesto para lograr nanoemulsiones dobles. Una de estas es la descrita por Hanson et al. [37] que propone el uso de bloques de copolipéptidos con estructura general poly $\left(\mathrm{L}-\mathrm{lysine}^{*} \mathrm{HBr}\right)_{\mathrm{x}}-\mathrm{b}-$ poly(racemic-leucine)y, $\mathrm{Kx}(\mathrm{rac}-\mathrm{L})_{\mathrm{y}}$ en donde el segmento hidrofílico corresponde a poly $\left(\mathrm{L}-1 \mathrm{ysine}{ }^{*} \mathrm{HBr}\right)_{\mathrm{x}(20-100}$ residuos) $\mathrm{y}$ el

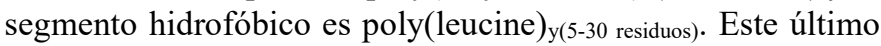
capaz de formar una estructura de varilla helicoidal que puede penetrar la fase oleosa e interaccionar con la fase acuosa W1 a través de su parte $\mathrm{Kx}(\mathrm{rac}-\mathrm{L})$. Facilitando la formación de nanoemulsiones $\mathrm{W} / \mathrm{O} / \mathrm{W}$. Aunque no tiene una aproximación nanotecnológica, Kim et al. [38] desarrollaron un proceso de doble emulsificación en una etapa basada en colisiones entre 
glóbulos formados, al colisionar consiguen interiorizar la fase continúa convirtiéndola en fase dispersa, de esta manera se crea una doble emulsión. La emulsión O/W/O se logra obtener mediante la fase continua compuesta por tolueno, un polímero liposoluble y partículas de sílica. A pesar del enfoque innovador descrito, se requieren diversos estudios para determinar cuáles son las condiciones que permiten obtener un tamaño nanométrico y estabilicen la formación de los glóbulos internos. Zhao et al. [39] siguiendo los ensayos descritos por Zhang et al. [40], fueron capaces de sintetizar una nanoemulsión doble mediante el uso de una cámara de alta presión a la cual suministraron $\mathrm{CO}_{2}$. Dichos experimentos indicaron que bajo ciertas condiciones de presión y concentración de surfactante iónico las moléculas de $\mathrm{H}_{2} \mathrm{O}$ y tensioactivo son capaces de ingresar y estabilizarse dentro de la fase oleosa quedando como nanoemulsión $\mathrm{W} / \mathrm{O} / \mathrm{W}$. Se describe este método como barato y sencillo para lograr la doble emulsificación. Requiere del diseño y construcción de un equipo como el utilizado por los autores. Si bien, se ha utilizado los tensioactivos Tritón X100, Pluronic P104 y CTAB para la síntesis de nanoemulsiones por alta presión, la estabilidad de estas es baja y se observa desestabilización total en 2 horas. Finalmente, se recomienda leer la interesante revisión publicada por Sheth et al. [3], quienes realizan una clasificación general de las nanoemulsiones dobles: emulsificación por secuencia, engullimiento, coemulsificación y emulsificación por separación de fases.

\section{OTROS FACTORES QUE INFLUYEN EN LA FORMACIÓN DE NANOEMULSIONES}

La formulación de nanoemulsiones simples $(\mathrm{W} / \mathrm{O}$ y $\mathrm{O} / \mathrm{W})$ y en consecuencia las nanoemulsiones dobles $(\mathrm{W} / \mathrm{O} / \mathrm{W}$ y $\mathrm{O} / \mathrm{W} / \mathrm{O})$ requiere tomar en cuenta diversos factores experimentales o de operación. Estos factores dependen del método de síntesis, la concentración de sus componentes, la incorporación de compuestos activos, entre otros. A continuación, se describen diferentes factores a considerar en la formulación de nanoemulsiones en general.

\section{A.Balance hidrofilico-lipofilico (HLB)}

El balance hidrofílico-lipofílico es un término desarrollado por William Griffin en 1949, definido como el balance entre tamaño y fuerza de la cabeza hidrofílica y lipofílica, a la cual se le puede asignar un valor numérico. El aporte o contribución de los diferentes grupos de la molécula indicarán su solubilidad y capacidad para formar emulsiones W/O u $\mathrm{O} / \mathrm{W}$ [41]. Posteriormente, diferentes autores contribuirían otras ecuaciones para relacionar el número HLB con otros grupos funcionales e incluso con surfactantes iónicos. En general un HLB entre 1.5-8 son surfactantes que permiten la formación de emulsiones $\mathrm{W} / \mathrm{O}$, los surfactantes con valores sobre 8 son útiles para formar emulsiones $\mathrm{O} / \mathrm{W}$ [42]. Otros autores han descrito nuevos métodos para el cálculo del número HLB los cuales pueden ser consultados en [42], [43], [44]-[45]. Estos incorporan diversos parámetros que el método HLB no toma en cuenta como la temperatura, salinidad, \% de las fases, estructurales, entre otros. A pesar de los esfuerzos realizados, el método HLB sigue estando vigente debido a que permite entender el proceso de emulsificación de manera simplificada.

\section{B.HLB y su relación en nanoemulsiones}

El método de producción junto a otros parámetros determina el tamaño de glóbulo, polidispersidad y estabilidad. $\mathrm{Lu}$ et al. [46] realizando estudios para la preparación y caracterización de nanoemulsiones $\mathrm{O} / \mathrm{W}$ incorporando aceite esencial de citral, logran determinar el HLB requerido, utilizando los emulsionantes Span 85 (HLB=1.8) y Brij 97 $($ HLB $=12)$. Variando el HLB entre 2 a 12 , se determinó que existe una relación lineal entre HLB y tamaño de glóbulo, obteniendo un tamaño mínimo para HLB 12. Sin embargo, se registró el aumento de tamaño de glóbulo. Asociado principalmente al método de producción y la alta energía aplicada, la nanoemulsión alcanza un periodo de maduración relacionada con la desestabilización Ostwald ripening.

La elección de surfactantes para nanoemulsiones del tipo W/O ó $\mathrm{O} / \mathrm{W}$ se realiza dependiendo de su afinidad por determinada fase. En el caso del tensioactivo hidrofílico Tween 80 debiese ser añadido en la fase acuosa, mientras que un tensioactivo hidrofóbico (e.g. Span 80) debe ser añadido en la fase oleosa [47]. Sin embargo, es posible añadir el surfactante hidrofílico en la fase oleosa incrementando la temperatura. A medida que el emulsionante se aproxima al punto de enturbiamiento ( $c p=$ cloud point $)$ comienza a tener solubilidad reversa y será más afín a la fase oleosa. Esta es una propiedad que se puede utilizar para incrementar la sinergia entre tensioactivos con afinidad opuesta. De este modo el HLB se hace manejable y mejora la estabilidad de la nanoemulsión final. La adición de otros compuestos como co-surfactantes, espesantes y/o un compuesto osmóticamente activo permite equilibrar fuerzas y evita desestabilizaciones debido a la presión osmótica [48].

\section{dobles}

C.Efectos energéticos en la formación de nanoemulsiones

Otro factor importante en la formación de nanoemulsiones doble es la energía suministrada para lograr la emulsificación. Dependiendo del método seleccionado, existirán diversos parámetros que se deben manejar u optimizar. En el caso de los métodos de alta energía como homogenización por ultrasonido, homogenización por alta presión y homogenización de alta velocidad puede ocurrir un efecto llamado sobreprocesamiento, en donde el tamaño de los glóbulos formados incrementa debido a desestabilizaciones asociadas a coalescencia y Ostwald ripening (principalmente por esta última) [49]-[50] Diversos autores mencionan que mayores tiempos de homogenización y una concentración adecuada de tensioactivo llevan a la obtención de nanoemulsiones con menor diámetro [51]-[52]. Sin embargo, en nanoemulsiones dobles, un mayor tiempo de homogenización no necesariamente indica menor tamaño de partícula y estabilidad. Tang \& Sivakumar. [53], en estudios de encapsulación de aspirina en una nanoemulsión doble 
mediante homogenización ultrasonido sugiere realizar la segunda emulsificación con menor amplitud y tiempo de homogenización para no romper el sistema. Bamba et al. [54] determinaron el efecto de diversas condiciones de homogenización (Microfluidizador y alta velocidad) sobre una nanoemulsión doble para la encapsulación de polifenoles y antocianinas. Los autores observaron que bajo ciertas condiciones de presión o velocidad, hay un incremento en el tamaño de glóbulo, asociado a la imposibilidad del emulsionante atenuar rápidamente el aumento de la tensión superficial y la presión de Laplace. Un incremento en la concentración de surfactante permite soportar la disminución del tamaño de glóbulo [55]. Aunque, una mayor concentración de surfactante es un parámetro que se debe evaluar dependiendo de la aplicabilidad de la nanoemulsión (e.g en cosmética una alta concentración de emulsionante puede causar irritación en la piel).

\section{PROCESO DE ESCALAMIENTO}

El proceso de escalamiento para nanoemulsiones dependerá del equipo y método utilizado para dicho fin. Si bien, en esencia los métodos de baja y alta energía son diferentes, mantienen en común la necesidad de una adecuada distribución de sus fases. Los métodos que requieren agitación mecánica van desde la emulsificación tradicional hasta los métodos de inversión de fases (temperatura y espontánea). La agitación mecánica sigue mecanismos generales para lograr una distribución. En este caso de la agitación mecánica es posible hacer uso de diversas relaciones provenientes del análisis de reactores agitados. Solè et al. [56] estudiaron el proceso de escalamiento para una nanoemulsión hecha mediante el método PIC (phase inversión composition). En primera instancia realizaron un análisis de dimensiones del recipiente. Respetando proporciones de altura y ancho, al igual que el tamaño del agitador. Posteriormente, se determinó estadísticamente si los factores velocidad de adición de la fase dispersa, velocidad lineal, tiempo total de adición y velocidad característica son significativos para diferentes formulaciones. Adicionalmente se propone el uso de la velocidad lineal característica como parámetro de escalamiento mostrando una buena relación entre tamaño de glóbulo y volumen de reacción. Se desestima el uso del número de Reynolds como parámetro debido a que el aumento de escala podría generar una velocidad angular excesiva. Otras relaciones de escalamiento pueden ser utilizadas, como la relación empírica entre número de Reynolds y el número de Newton o número de poder, este último característico para cada tipo de agitador. Sin embargo, se debe tener en consideración el régimen laminar o turbulento, y el tipo de fluido, Newtoniano o no Newtoniano, este último característico en formulaciones cosméticas y alimentarias. En base a lo anterior, las relaciones de potencia necesaria para la agitación pueden ser modificadas, como la propuesta por Metzner-Otto [57]. Un ejemplo de esto es lo descrito por James et al. [58], en donde desarrolla ecuaciones empíricas de escalamiento para regímenes laminares y turbulentos logrados mediante un agitador rotor-estator marca Silverson. En el caso de las nanoemulsiones preparadas mediante homogenización por ultrasonido, los factores operacionales que dominan el proceso son el tiempo de homogenización (expresado como tasa de procesamiento o $\mathrm{mL}$ emulsión/minuto) para operaciones continuas, amplitud, potencia y configuración de la sonda del equipo [59]. Además de lograr una formulación ideal en términos de proporciones de fases continua, dispersa y concentración de surfactante, es importante determinar las condiciones de procesamiento que permiten un tamaño nanométrico de los glóbulos sin causar sobreprocesamiento de estos, lo cual puede ser evidenciado al observar un aumento en el tamaño de glóbulo y una disminución en la capacidad de encapsulación [60]. Khadem \& Sheibat [61], desarrollaron ecuaciones de modelamiento para predecir el tamaño y capacidad de encapsulación en emulsiones dobles siguiendo a las aproximaciones de Taylor, Hinze y las modificaciones propuestas por Gupta et al. [32]. De igual manera que en nanoemulsiones simples, un aumento en el tiempo de procesamiento genera una menor capacidad de encapsulación debido al rompimiento de los glóbulos. Si bien, se describen buenas correlaciones para emulsiones micrométricas, en nanoemulsiones dobles los tamaños de glóbulo se mantienen $<500 \mathrm{~nm}$, esto genera la necesidad de una mayor cantidad de energía que puede provocar la desestabilización de la nanoemulsión interior. Lo descrito por Khadem \& Sheibat [61] es una buena aproximación para explicar el proceso de doble emulsificación por ultrasonido, pero es necesario un mayor análisis para explicar la síntesis de nanoemulsiones dobles sub-micrométricas. Otras estrategias utilizadas para lograr nanoemulsiones son las referentes al uso de homogenización por alta presión, microfluidizador y microfluídica. Las dos primeras utilizan altas presiones (50$350 \mathrm{MPa}$ ) y una estructura determinada por donde pasa una emulsión gruesa. El gradiente de presión genera una emulsión fina de glóbulo pequeño que puede ser controlado mediante diferentes ciclos de procesamiento. Se ha descrito el uso de estas estrategias para formular emulsiones dobles. Sin embargo, no se recomienda para producir nanoemulsiones dobles, a fin de evitar la alta energía y sobreprocesamiento de estas, cuyo principal efecto es la desestabilización y rompimiento de los glóbulos evidenciado a través de un aumento en el tamaño de glóbulo [62].

\section{APLICACIONES DE LAS NANOEMULSIONES DOBLES}

Las nanoemulsiones y la encapsulación de compuestos activos dentro de un glóbulo permiten la protección de estos frente a diversos factores ambientales como radiación UV, temperatura, humedad, agentes oxidantes, entre otros. Además de mejorar la biodisponibilidad debido a la disminución en el tamaño de partícula y aumento en el área de contacto [63]. En particular las nanoemulsiones dobles permiten la incorporación de compuestos activos de diversa naturaleza química. El campo de aplicación es variado y comprende áreas como la cosmética, farmacéutica, medicina de diagnóstico y tratamiento, industrial, etc [3]. 


\section{A. Alimentos}

El desarrollo de nanoemulsiones en la industria de alimentos está destinado a evitar la degradación de los compuestos activos y aumentar la biodisponibilidad de estos. Además de proteger dichos compuestos en su paso por el aparato digestivo. La encapsulación se realiza principalmente para minerales, proteínas, aminoácidos, pigmentos, endulzantes, prebióticos. Sin embargo, no es posible para probióticos, debido al tamaño celular [64]-[65]. Un caso de estudio interesante es la amplia literatura con respecto a incrementar la biodisponibilidad de la curcumina y su concentración en la sangre. Adicionalmente, se han desarrollado varios métodos para este fin, que no solo incluye a nanoemulsiones, sino también conjugados poliméricos, liposomas, emulsiones Pickering, entre otros [66]. Una alternativa para complementar compuestos lipofílicos como la curcumina con compuestos hidrofílicos es el desarrollo de nanoemulsiones dobles. Adtiya et al. [67] desarrollaron una emulsión doble que en su primera fase dispersa (acuosa) contenía catequina (antioxidante polifenólico), gelatina (espesante), cloruro de sodio y ácido ascórbico, estos compuestos fueron inmersos en la fase oleosa compuesta de aceite de oliva, curcumina y el tensioactivo hidrofóbico poliglicerol polirricinoleato (PGPR, HLB=0.6). Posteriormente, utilizando homogenización de ultrasonido, se añadió la primera emulsión en la fase dispersa final, compuesta por $\mathrm{H}_{2} \mathrm{O}$ y el surfactante Tween $80(1 \% \mathrm{v} / \mathrm{v})$. Sin embargo, el tamaño del glóbulo formado se estimó en $6.7 \mu \mathrm{m}$ $(6700 \mathrm{~nm})$. Una estrategia para la disminución del tamaño de glóbulo es la adición de una mayor cantidad de tensioactivo [68]. Gharehbeglou et al. [36], consiguieron nanoemulsiones dobles cargadas con oleuropeína, un Polifenol presente en la pulpa de aceitunas verdes y hojas de olivo. La oleuropeína fue añadida en la nanoemulsión doble siguiendo el método de dos pasos, para lo cual se utilizó una fase oleosa compuesta por aceite de soja junto al emulsionante Span 80. La nanoemulsión doble final se obtuvo mediante el uso de WPC (whey protein concentrate), el espesante pectina y homogenización por ultrasonido durante 10 minutos. Siguiendo un diseño experimental se obtuvo un glóbulo de $191.1 \mathrm{~nm}$. Mehrnia et al. [69] desarrollaron una nanoemulsión doble para la encapsulación del compuesto bioactivo crocina, carotenoide proveniente de la especia azafrán, sensible a diversas condiciones de almacenamiento. Mediante el uso del emulsionante PGPR y aceite de oliva se pudo realizar la nanoemulsión primaria W/O. La nanoemulsión final W/O/W consistió en WPC y goma arábica. Recurriendo a métodos de alta energía se generó una emulsión gruesa mediante homogenización por rotor-estator y homogenización por alta presión a temperatura ambiente. Una revisión más detalla de emulsiones y nanoemulsiones dobles destinadas a la industria de alimentos se puede encontrar en Mohammad et al [65].

\section{B. Cosmética}

Los cosméticos han tenido un rol fundamental en la historia humana. Si bien, las grandes empresas de cosméticos se fundan a partir del siglo XX, ya las culturas mesopotámicas $\mathrm{y}$ egipcias utilizaban ungüentos con fines religiosos, terapéuticos y estéticos. Estas costumbres luego son llevadas a Grecia y Roma, en donde crean sus propias mezclas destinadas al cuidado de la piel [70].

Actualmente se estima que la industria cosmética obtiene ingresos aproximados de 537 billones de dólares, por productos destinados al cuidado de la piel y protectores solares, cabello, maquillaje, fragancias [71]-[72]. Las principales marcas que lideran el mercado son L'Oreal, Unilever, The Estée Lauder, Procter \& Gamble, Shiseido, Coty, LVMH, Beiersdorf, entre otras [73]. Se han desarrollado diversos productos con base nanotecnológica, los cuales incluyen nanopartículas lipídicas sólidas, niosomas, liposomas, dendrímeros, polímeros, nanopartículas en general [74]. L'Oreal es una de las principales empresas que invierten en tecnología y desarrolla productos con base nanotecnológica. Si bien en el año 2004 invertía aproximadamente $\$ 600$ millones de dólares, en la actualidad invierte más de 1 billón de dólares en ciencia y tecnología [75]. Esta compañía fue la primera en lanzar un producto que incluía "nanosomas" o liposomas para la encapsulación de Pro-retinol A. En la Tabla II, se observan patentes registradas para nanoemulsiones dobles destinadas a la industria cosmética.

\section{Farmacéutica y Biomedicina}

Las nanoemulsiones destinadas a la industria farmacéutica y aplicaciones biomédicas tienen como objetivo la incorporación de compuestos activos lipofílicos e hidrofílicos en estas matrices y la protección de estos de factores ambientales. Además de promover la liberación controlada de $y$ el incremento de la biodisponibilidad. Lo anterior es conocido como drug delivery, y es utilizada en el área oftalmológica, oral, tópica, transdérmica, intranasal y parenteral [76] (Tabla III). Dentro del desarrollo de este tipo de nanoemulsiones es esencial el uso adecuado de emulsionante y/o otros componentes, de modo que no genere daño celular y disrupción de la membrana celular [77]-[78].

Si bien, generalmente, se prefiere el uso de emulsionantes no iónicos, también es posible realizar emulsiones nanométricas con emulsionantes iónicos con bajos efectos celulares [79]. Existen diversos métodos para comprobar la seguridad de estos preparados y que se corresponden con su potencial uso. Por ejemplo, los ensayos de hemolisis son útiles cuando la nanoemulsión es de uso parenteral. En particular, el programa nacional de toxicología de los Estados Unidos dependiente de National Institute of Health, actualiza regularmente el listado de métodos alternativos (no animal testing) aceptados por diversos organismos como la FDA (Food and Drug Administration), USDA (United States Department of Agriculture) e ICCVAM (Interagency Coordinating Committee on the Validation of Alternative Methods). Esta última colabora con la OECD (Organisation for Economic Co-operation and Development) para la validación y utilización de los métodos en los países miembro [80]. En particular, la FDA regula todo producto o actividad 
relacionada a la nanotecnología, esto incluye productos o de la medicina [81]. procedimientos destinados a su uso en humanos y la práctica

TABLA II

PATENTES PARA NANOEMULSIONES DOBLES DESTINADAS A COSMÉTICA

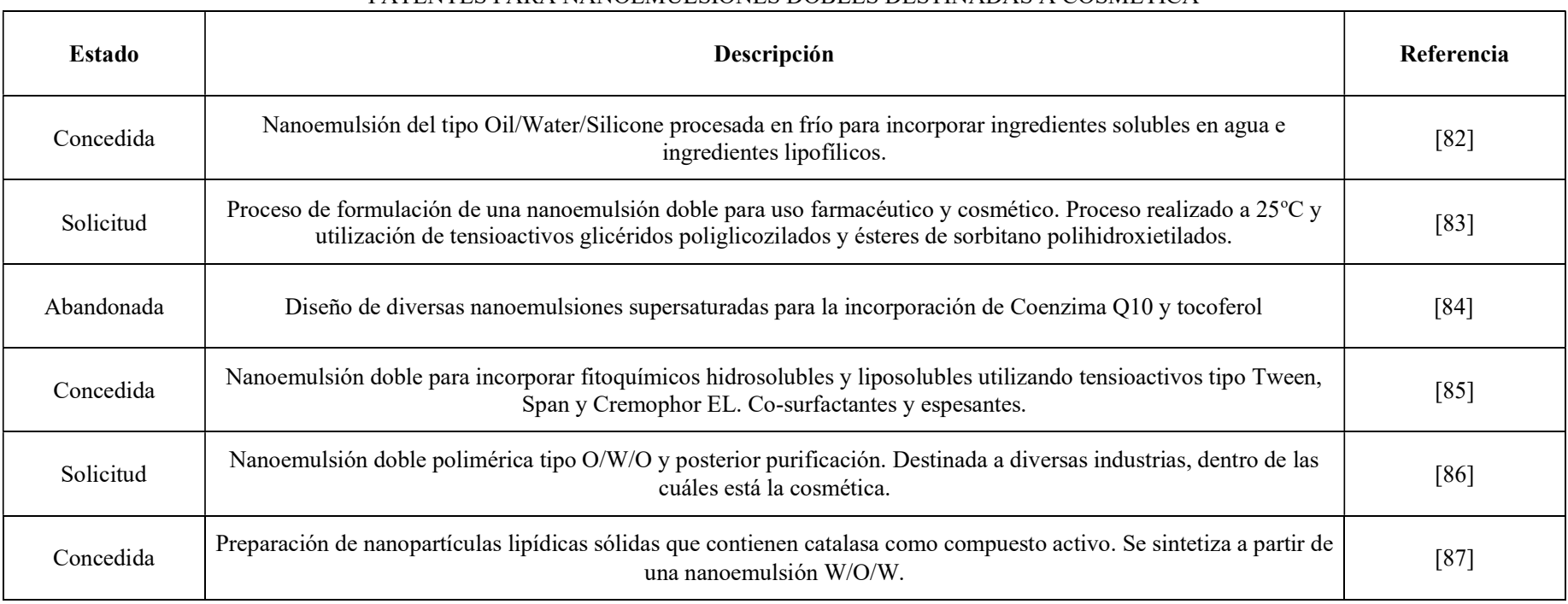

TABLA III

NANOEMULSIONES DOBLES DESTINADAS AL ÁREA FARMACÉUTICA Y BIOMÉDICA

\begin{tabular}{|c|c|c|c|}
\hline Tamaño (nm) & Compuesto & Descripción & Referencia \\
\hline 257 & $\begin{array}{c}\mathrm{mAb} \text { anti-EGFR, mitomicina y verde } \\
\text { indocianina }\end{array}$ & $\begin{array}{l}\text { Encapsulación de compuestos activos para tratamiento de cáncer de vejiga } \\
\text { mediante fototerapia. }\end{array}$ & [88] \\
\hline 25 & 5-fluoracil & $\begin{array}{c}\text { Nanoemulsión doble para la encapsulación de un compuesto } \\
\text { quimiopreventivo. }\end{array}$ & [90] \\
\hline $174.3-184.5$ & Gadolinium (III) & $\begin{array}{c}\text { Nanoemulsión doble que contiene Gadolinium (III) como contraste para } \\
\text { resonancia magnética. }\end{array}$ & {$[91]$} \\
\hline 500 & Insulina & Nanoemulsión doble-polimérica para la encapsulación de insulina & [92] \\
\hline 160 & Celecoxib/Cellvue & $\begin{array}{c}\text { Incorporación de un inhibidor de } \mathrm{COX}-2 \text { y prueba fluorescente en una } \\
\text { nanoemulsión } \mathrm{O} / \mathrm{O} / \mathrm{W} \text {. }\end{array}$ & [93] \\
\hline
\end{tabular}

\section{CONCLUSIONES}

Existe una vasta y compleja literatura para explicar la formación de nanoemulsiones. De igual forma se han publicado un sin número de artículos evidenciando las ventajas de los sistemas nanoemulsionados. Si bien, no es el único método con enfoque nanotecnológico para aumentar la disponibilidad de diversos compuestos, este es un proceso fácil de entender y de poder implementar gracias a la amplia disponibilidad de agentes tensioactivos y equipamientos disponibles, e incluso sin equipamiento sofisticado se puede realizar si se recurre a métodos como emulsificación espontanea o PIT. Por otra parte, el desarrollo de las nanoemulsiones dobles entrega una versatilidad aún mayor al mundo de la emulsificación. Esto permite incorporar de diferentes maneras compuestos químicos que no podrían unirse mediante una nanoemulsión normal o que sí podrían, pero uno de ellos no se encontraría en el glóbulo nanométrico. El desarrollo de este tipo de nanoemulsiones requiere un análisis más fino debido a que la fase dispersa corresponde a una nanoemulsión W/O que puede ser desestabilizada si no se optimizan los factores imperantes dependiendo del método utilizado (alta energía). Sin embargo, es posible realizar formulaciones que combinen métodos de alta y baja energía para evitar el sobreprocesamiento y rotura de los glóbulos internos. 


\section{AGRADECIMIENTOS}

La elaboración de esta revisión se enmarca en el desarrollo del proyecto FONDEF VIU18E0030 "Nanoemulsión con propiedades biomédicas a base de los compuestos bioactivos curcumina y micosporina para aplicaciones en el ámbito cosmético y de productos naturales", el cual se financia mediante el programa Valorización de la Investigación en la Universidad (VIU) a través del Fondo de Fomento al Desarrollo Científico y Tecnológico (FONDEF) perteneciente a la Agencia Nacional de Investigación y Desarrollo (ANID), Gobierno de Chile.

\section{REFERENCIAS}

[1] F. Goodarzi, S. Zendehboudi, "A Comprehensive Review on Emulsions and Emulsion Stability in Chemical and Energy Industries", The Canadian Journal of Chemical Engineering, vol. 97, no. 1, pp. 281-309, 2018.

[2] N.A.N. Azmi, A.A.M. Elgharbawy, S.R. Motlagh, N. Samsudin, H.M. Salleh, "Nanoemulsions: Factory for Food, Pharmaceutical and Cosmetics", Processes, vol. 7, no. 9, pp. 617, 2019.

[3] T. Sheth, S. Seshadri, T. Prileszky, M. E. Helgeson, "Multiple nanoemulsions", Nature Reviews Materials, vol. 5, pp. 214-228, 2020.

[4] S.N. Kale, S.L. D, "Emulsion Micro emulsion and Nano Emulsion: A Review", Systematic Reviews in Pharmacy, vol. 8, no. 1, pp. 39-47, 2017.

[5] N. Anton, T.F. Vandamme, "The universality of low-energy nanoemulsification", International Journal oh Pharmaceutics, vol. 377, no.12, pp.142-147, 2009.

[6] N. Anton, T.F. Vandamme, "Nano-emulsions and Micro-emulsions: Clarifications of the Critical Differemces", Pharmaceutical Research, vol. 28, no. 5, pp.978-985, 2010.

[7] P. Karthik, C. Anandharamakrishnan, "Fabrication of a nutrient delivery system of docosahexaenoic acid nanoemulsions via high energy techniques", vol. 6, no. 5, pp.3501-3513, 2016.

[8] Dammak, R. A. de Carvalho, C. S. F. Trindade, R. V. Lourenço, P. J. do Amaral, "Properties of active gelatin films incorporated with rutinloaded nanoemulsions", International Journal of Biological Macromolecules, vol. 98, pp.39-49, 2017.

[9] G. Lefebvre, J. Riou, G. Bastiat, E. Roger, K. Frombach, J. C. Gimel, P. Saulnier, B. Calvignac, "Spontaneous nano-emulsification: Process optimization and modeling for the prediction of the nanoemulsion's size and polydispersity", International Journal of Pharmaceutics, vol. 534, no. $1-2,2017$.

[10] T. Mehmood, A. Ahmed, A. Ahmad, M. S. Ahmad, M. A. S, "Optimization of mixed surfactants-based $\beta$-carotene nanoemulsions using response surface methodology: An ultrasonic homogenization approach", Food Chemistry, vol. 253, pp. 179-184, 2018.

[11] J. McClements, "Nanoemulsions versus microemulsions: terminology, differences and similarities", Soft Matter, vol. 8, no. 6, pp.1719-1729, 2012.

[12] R. J. Hunter. Foundations of colloid science, vol II. 1st ed. Oxford: Oxford University Press, 1989.

[13] T. F. Tadros. "Emulsion Formation, Stability and Rheology" in Emulsion Formation and Stability, T. F. Tadros, 1st ed. Weinheim: Wiley-VCH, pp.1-75, 2013.

[14] G. G. Láng, "Basic interfacial thermodynamics and related mathematical background", ChemTexts, vol. 1, no.16, pp.1-17, 2015.

[15] S. Rawal, M. Patel, "Lipid nanoparticulate systems: Modern versatile drug carriers" in Lipid Nanocarriers for Drug Targeting, 1st ed. Elsevier William Andrew, pp.49-138, 2018.

[16] S. Obravić, M. Poša, "Th influence of the structure of selected Brij and Tween homologues on the thermodynamic stability of their binary mixed micelles", Journal of Chemical Thermodynamics, vol. 110, pp.41-50, 2017.

[17] S. Slomkowski, J. Alemán, J. Gilbert, G. Hess, K. Horie, R. Jones, "Terminology of polymers and polymerization process in dispersed systems (IUPAC Recommendations 2011), Pure and Applied Chemistry, vol.83, no.12, pp.2229-2259, 2011.

[18] Solans, I. Solé, "Nano-emulsions: Formation by low-energy methods", Current Opinion in Colloid \& Interface Science, vol.17, no.5, pp.246$254,2012$.
[19] C. Lovelyn, A. Attama, "Current state of Nanoemulsion in Drug Delivery", Journal of Biomaterials and Nanobiotechnology, vol. 2, no.5, pp.629-639, 2011.

[20] S. Nemichand, S. Laxman, "Emulsion Micro Emulsion and Nano Emulsion: A Review", Systemic Reviews in Pharmacy, vol.8, no.1, pp.39-47, 2017.

[21] Acosta, E. Szekeres, J. Harwell, B. Grady, D. Sabatini, "Morphology of ionic microemulsions: comparison of SANS studies and the net-average curvature (NAC) model", Soft Matter, vol.5, no.3, pp.551-561, 2008.

[22] S. Kiran, E. Acosta, "Predicting the morphology and viscosity of Microemulsions Using the HLD-NAC Model”, Industrial \& Engineering Chemistry Research, vol.49, no.7, pp.3424-3432, 2010.

[23] B. Lukanov, A. Firoozabadi, "Molecular Thermodynamic Modeling of Reverse Micelles and Water-in-oil Miroemulsions", Langmuir, vol.32, no.13, pp.3100-3109, 2016.

[24] M. Turchi, Q. Cai, G. Lian, "In silico Prediction of the Thermodynamic Equilibrium of Solute Partition in Multiphase Complex Fluids: A Case Study of Oil-Water Microemulsion", Langmuir, vol.35, pp.1085510865, 2019.

[25] K. Motomura, J. Baret, "Thermodynamic Consideration of Microemulsions", Journal of Colloid and Interface Science, vol.91, no.2, pp.391-399, 1982.

[26] Tartaro, H. Mateos, D. Schirone, R. Angelico, G. Palazzo, "Microemulsion Microstructure(s): A Tutorial Review", Nanomaterials, vol.10, no.9, pp.1657, 2020.

[27] Ruckenstein, "Phase Inversion Temperatures of Macro- and Microemulsions", Langmuir, vol.13, no.9, pp.2494-2497, 1997.

[28] Kaptay, "Partial Surface Tension of Components of a Solution", Langmuir, vol.31, no.21, pp.5796-5804, 2015.

[29] Kaptay, "On the Negative Surface Tension of Solutions and on Spontaneous Emulsification", Langmuir, vol.33, no.40, pp.10550-10560, 2017.

[30] S. Ding, N. Anton, S. Akram, M. Rafik, H. Anton, A. Klymchenko, W. $\mathrm{Yu}, \mathrm{T}$. Vandamme, C. Serra, "A new method for the formulation of double nanoemulsions", Soft Matter, vol.13, pp.1660-1669, 2017.

[31] Håkansson, M. Rayner, "General Principles of Nanoemulsion Formation by High-Energy Mechanical Methods" in Nanoemulsions: Formulation, Applications and Characterization, S. Mahdi, D. McClements, 1 ed., Elsevier, pp.103-139, 2018.

[32] Gupta, H. Burak, T. Hatton, P. Doyle, "Controlling and predicting droplet size of nanoemulsions: scaling relating with experimental validation", Soft Matter, vol.12, pp.1452-1458, 2016.

[33] Håkansson, "Fabrication of Nanoemulsions by High-Pressure Valve Homogenization" in Nanoemulsions: Formulation, Applications and Characterization, S. Mahdi, D. McClements, 1 ed., Elsevier, pp.175-206, 2018.

[34] J. Davies, "A physical interpretation of Drop Sizes In Homogenizers and Agitated Tanks, Including the Dispersion of Viscous Oils", Chemical Engineering Science, vol.42, no.5, pp.1683-1692, 2010.

[35] M. Kanouni, H. Rosano, N. Naouli, "Preparation of a stable double emulsion (W1/O/W2): role of the interfacial films on the stability of the system", Advances in Colloid and Interface Science, vol.99, no.3, pp.339-354, 2002.

[36] P. Gharehbeglou, S. Jafari, A. Homayouni, H. Hamishekar, H. Mirzaei, "Fabrication of double W1/O/W2 nano-emulsions loaded with oleuropein in the internal phase (W1) and evaluation of their release rate", Food Hydrocolloids, vol.89, pp.44-55, 2019.

[37] J. Hanson, C. Chang, S. Graves, Z. Li, T. Mason, T. Deming, "Nanoscale double emulsions stabilized by single-component block copolypeptides", Nature Letters, vol.455, pp.85-88, 2008.

[38] S. Kim, K. Kim. S. Choi, "Controllable one-step double emulsion formation via phase inversion", Soft Matter, vol.14, no.7, pp.1094-1099, 2018.

[39] Y. Zhao, J. Zhang, Q. Wang, J. Li, B. Han, "Water-in-oil-in-water double nanoemulsion induced by CO2", Physical Chemistry Chemical Physics, vol.13, pp.684-689, 2010.

[40] R. Zhang, J. Liu, J. He, B. Han, Z. Liu, T. Jiang, W. Wu, L. Rong, H. Zhao, B. Dong, G. Hu, "Compressed Ethylene-Assisted Formation of the Reverse Micelle of PEO-PPO-PEO Copolymer", Macromolecules, vol.36, pp.1289-1294, 2003.

[41] R. P, M. Taurozzi, C. Bregni, "Some considerations about the hydrophilic-lipophilic balance system", International Journal of Pharmaceutics, vol.356, pp.44-51, 2008.

[42] Y. Yamashita, K. Sakamoto, "Hydrophilic-Lipophilic Balance (HLB) Classical Indexation and Novel Indexation of Surfactant" in 
Encyclopedia of Biocolloid and Biointerface Science, 2 Volume Set, $\mathrm{H}$. Ohshima, 1st ed., Jhon Wiley \& Sons, pp-570-574, 2016.

[43] K. Corin, C. O’Connor, "A proposal use excess Gibbs energy rather than HLB number as an indicator of the hydrophilic-lipophilic as an indicator of the hydrophilic-lipophilic behavior of surfactants", Mineral Engineering, vol.58, pp.17-21, 2014.

[44] T. Nguyen, C. Morgan, L. Poindexter, J. Fernandez, "Application of the Hydrophilic-Lipophilic Deviation Concept to Surfactant characterization and Surfactant Selection for Enhanced Oil Recovery", Journal of Surfactants and Detergents, vol. 22, no.5, pp.983-999, 2019.

[45] J. Wu, Y. Dabros, H. Hamza, "Development of a method for measurement of relative solubility of nonionic surfactants", Colloid and Surfaces A: Physicochemical Engineering Aspects, vol.232, pp.229-237, 2004.

[46] W. Lu, D. Huang, C. Wang, C. Yeh, J. Tsai, Y. Huang, P. Li, "Preparation, characterization and antimicrobial activity of nanoemulsions incorporating citral essential oil", Journal of Food and Drug Analysis, vol.26, no.1, pp.82-89, 2018.

[47] W. Chong, C. Tan, Y. Cheah, A. Lajis, M. Habi, S. Kanagaratnam, O. Lai, "Optimization of process parameters in preparation of tocotrienolrich palm oil-based nanoemulsions stabilized by Tween 80 -Span 80 using response surface methodology", PLoS One, vol.13, no.8, e0202771, 2018.

[48] Juárez, J. García, "Structures similar to lipid emulsions and liposomes Dipalmitoylphophatidylcholine, cholesterol, Tween20-Span20 or Tween80-Span80 in aqueous media", Journal of Liposome Research, vol.27, no.2, pp.139-150, 2017.

[49] N. Leister, H. Karbstein, "Evaluating the stability of Double emulsionsA review of the Measurement Techniques for the Systemic Investigation of Instability Mechanisms", vol.4, no.8, pp.1-18, 2020.

[50] M. Koroleva, T. Nagovitsina, E. Yurtov, "Nanoemulsions stabilized by non-ionic surfactants: stability and degradation mechanism", Physical Chemistry Chemical Physics, vol.20, no.15, pp.10369-1037, 2018.

[51] T. Delmas, H. Piraux, A. Couffin, I. Texier, F. Vinet, P. Poulin, M. Cates, J. Bibette, "How to Prepare and Stabilize Very Small Nanoemulsions", Langmuir, vol.27, no.5, pp.1683-1692, 2011.

[52] S. Uluata, E. Decker, D. McClements, "Optimization of Nanoemulsion Fabricating Using Microfluidization: Role of Surfactant concentration and Stability", Food Biophysics, vol.11, no.1, pp.52-59, 2015.

[53] S. Tang, M. Sivakumar, "Design and evaluation of aspirin-loaded waterin-oil-in-water submicron multiple emulsions generated using two-stage ultrasonic cavitational emulsification technique", Asia-Pacific Journal of Chemical Engineering, vol.7, pp.145-156, 2012.

[54] Bamba, J. Shi, C. Tranchant, S. Xue, C. Forney, L. Lim, W. X, G. Xu, "Co-encapsulation of Polyphenols and Anthocyanins from Blueberry Pomace by Double emulsion Stabilized by Whey Proteins: Effect of Homogenization Parameters", Molecules, vol.23, no.10, pp.2525, 2018.

[55] J. Carpenter, V. Saharan, "Ultrasonic assisted formation and stability of mustard ion in water nanoemulsion: Effect of process parameters and their optimization", Ultrasonic Sonochemistry, vol.35, pp.422-430, 2017.

[56] Solè, C. Pey, A. Maestro, C. González, M. Porras, C. Solans, J. Gutiérrez, "Nanoemulsions prepared by the phase inversion composition method: Preparation viariables and scale up", Journal of Colloid and Interface Science, vol.344, pp.417-423, 2010.

[57] Archard, M. Marouche, H. Boisson, "Hydrodynamics and Metzner-Otto correlation in stirred vessels for yield stress fluids", Chemical Engineering Journal, vol.125, no.1, pp.15-24, 2006.

[58] J. James, M. Cooke, L. Trinh, R. Hou, P. Martin, T. Kowalski, T. Rodgers, "Scale-up of batch rotor stator mixers. Part 1-power constants", Chemical Engineering Research and Design, vol.124, pp.313-320, 2017.

[59] Peshkoysky, S. Peshkoysky, S. Bystryak, "Scalable high-power ultrasonic technology for the production of translucent nanoemulsions", Chemical Engineering and Processing, vol.69, pp.77-82, 2013.

[60] Teng, M. He, J. Xu, F. Chen, C. Wu, Z. Wang, Y. Li, "Effect of ultrasonication on the stability and storage of soy protein isolatephosphatidylcholine nanoemulsions", Scientific Reports, vol.10, no.1, pp.14010, 2020.

[61] Khadem, N. Sheibat, "Theoretical and experimental investigations of double emulsion preparation by ultrasonication", Industrial \& Engineering Chemistry Research, vol.58, pp.8220-8230, 2019.

[62] S. Ding, C. Serra, T. Vandamme, W. Yu, N. Anton, "Double emulsions prepared by two-step emulsification: History, state-of-the-art and perspective", Journal of Controlled Release, vol.295, pp.31-49, 2019.
[63] Y. Singh, J. Meher, K. Raval, F. Khan, A. Chaurasia, N. Jain, M. Chourasia, "Nanoemulsion: Concepts, development and applications in drug delivery", Journal of Controlled Release, vol.252, pp.28-49, 2017.

[64] P. Eslami, L. Davarpanah, F. Vahabzadeh, "Encapsulating role of $\beta$ cyclodextrin in formation of Pickering water-in-oil-in-water (W1/O/W2) double emulsions containing Lactobacillus dellbrueckii”, Food Hydrocolloids, vol.64, pp.133-148, 2017.

[65] N. Mohammad, H. Saberian, S. Jafari, "Encapsulation of food ingredients by double nanoemulsions" in Lipid-Based Nanostructures for Food Encapsulation Purposes: Volume 2, S. Jafari, 1st ed., Academic Press, pp.89-128, 2019.

[66] Araiza, M. Akhtar, A. Sarkar, "Recent advances in emulsion-based delivery approaches for curcumin: From encapsulation to bioaccessibility", Trends in Food Science \& Technology, vol.71, pp.155$169,2018$.

[67] N. Aditya, S. Aditya, H. Yang, H. Kim, S. Park, S. Ko, "Co-delivery of hydrophobic curcumin and hydrophilic catechin by a water-in-oil-inwater double emulsion", Food Chemistry, vol.173, pp.7-13, 2015.

[68] T. Chuacharoen, S. Prasongsuk, C. Sabliov, "Effect of Surfactant Concentrations on Physicochemical Properties and Functionality of Curcumin Nanoemulsions Under Conditions Relevant to Commercial Utilization", Molecules, vol.24, no.15, pp.2744, 2019.

[69] M. Mehrnia, S. Jafari, B. Makhmal, Y. Maghsoudlou, "Rheological and release properties of double nano-emulsions containing crocin prepared with Angum gum, Arabic gum and whey protein", Food Hydrocolloids, vol.66, pp.259-267, 2017.

[70] F. González, L. Bravo, "History and present of skin care products, cosmetics and fragances. Especially those derived from plants", Ars Pharmaceutica, vol.58, no.1, pp.5-12, 2017.

[71] Business Insider, "Beauty has blown up to be a 532 billion industry analysts say that these 4 trends will make it even bigger", August 2020. [Online]. Available: https://www.businessinsider.com/beautymultibillion-industry-trends-future-2019-7

[72] Statista, "Value of the cosmetics market worldwide from 2018 to 2025", August $2020 . \quad$ [Online]. Available: https://www.statista.com/statistics/585522/global-value-cosmeticsmarket/

[73] Beauty Packaging, "Top 20 Global Beauty Companies", August 2020. [Online]. https://www.beautypackaging.com/heaps/view/6459/1

[74] S. Kaul, N. Gulati, D. Verma, S. Mukherjee, U. Nagaich, "Role of Nanotechnology in Cosmeceuticals: A Review of Recent Advances", Journal of Pharmaceutics, vol.2018, pp.1-19, 2018

[75] L'Oreal, "Beauty Research \& Innovation", August 2020. [Online]. Available: $\quad$ https://www.loreal.com/en/beauty-science-andtechnology/beauty-research-and-innovation

[76] R. Patel, M. Patel, S. Thakore, B. Patel, "Nanoemulsion as a valuable nanostructure platform for pharmaceutical drug delivery" in Nano-and Microscale Drug Delivery Systems: Design and Fabrication, A. Grumezscu, Cambridge, pp-321-341, 2017.

[77] P. Rocha, M. Ferrari, M. Maruno, O. Souza, "In vitro and In vivo Evaluation of Nanoemulsion Containing Vegetable Extracts", Cosmetics, vol.4, no.32, pp.1-13, 2017.

[78] S. Nunes, P. Quatrin, M. Rorato, K. Nascimento, R. Wagner, B. Klein, R. Vianna, O. Ferreira, "Evaluation of Stability and In vitro security of Nanoemulsions Containing Eucalyptus globulus Oil", BioMed Research International, vol.2017, pp.1-10, 2017.

[79] S. Lamaisakul, A. Tantituvanont, V. Lipipun, G. Ritthidej, "Development of novel cationic microemulsion as parenteral adjuvant for influenza vaccine", Asian Journal of Pharmaceutical Sciences, vol.15, no.5, pp.591-604, 2020.

[80] National Toxicology Program, "Alternative Methods Accepted by US Agencies", August 2020. [Online]. Available: https://ntp.niehs.nih.gov/whatwestudy/niceatm/acceptmethods/index.html

[81] Food And Drug Administration, "Nanotechnology Programs at FDA", August 2020. [Online]. Available: https://www.fda.gov/scienceresearch/science-and-research-special-topics/nanotechnology-programsfda

[82] M. Maidel Da Luz, R. Vecchi, R. Knapik, "Concentrated composition for cosmetic and/or dermatological treatment for facial application with anti-aging activity", BRPI1 105152A2, June 13, 2017.

[83] Brossard, S. Crauste, S. Muhamad, P. Odou, M. Blanco, A. Hermoso, "Method for making a formulation and use thereof for administering polar drugs", WO20100667035A1, June 17, 2010. 
[84] Belser, C. Liechti, F. Suter, F. Zülli, "Zubereitung Bestehend aus mindestens zwei Nanoemulsionen", EP1516662A1, March 03, 2005.

[85] S. Gunzburg, "Methods to create a double nanoemulsion for transdermal delivery of hydrophilic and hydrophobic phytochemical active ingredients", AU2018101231A4, September 27, 2018.

[86] M. Helgeson, S. Mitragotri, M. Zhang, M. Nowak, P. Malo de Molina, "Nanoscale multiple emulsions and nanoparticles", April 04, 2019.

[87] Jianhua, C. Yan, W. Xingguo, "Preparation method of catalase solid lipid nanoparticles preparation", December 14, 2011.

[88] Y. Lee, Y. Lin, “Anti-EGFR Indocyanine Green-Mitomycin C-Loaded Perfluorocarbon double Nanoemulsion: A novel Nanostructure for Targeted Photochemotherapy of Bladder Cancer Cells", Nanomaterials, vol.8, no.283, pp.1-18, 2018.

[89] Schwarz, V. Klang, S. Karall, D. Mahrhauser, G. Resch, C. Valenta, "Optimisation of multiple $\mathrm{W} / \mathrm{O} / \mathrm{W}$ nanoemulsions for dermal delivery of acyclovir", International Journal of Pharmaceutics, vol.435, pp.69-75, 2012.

[90] Shakeel, N. Haq, A. Al-Dhfyan, F. Alanazi, I. Alsarra, "Double W/O/W nanoemulsion of 5-fluorouracil for self-nanoemulsifying drug delivery system", Journal of Molecular Liquids, vol.200, pp.183-190, 2014.

[91] E. Sigward, Y. Corvis, B. Doan, K. Kindsiko, J. Seguin, D. Scherman, D. Brossard, N. Mignet, P. Espeau, S. Manciet, "Preparation and Evaluation of Multiple Nanoemulsions Containing Gadolinium (III) Chelate as a Potential Magnetic Resonance Imaging (MRI) Contrast Agent", Pharmaceutical Research, vol.32, no.9, pp.2983-2994, 2014.

[92] Li, J. Qi, Y. Xie, X. Zhang, S. Hu, Y. Xu, Y. Lu, W. Wu, "Nanoemulsions coated with alginate/chitosan as oral insulin delivery systems: preparation, characterization, and hypoglycemic effect in rats", International Journal of Nanomedicine, vol.8, pp.23-32, 2012.

[93] S. Patel, Y. Zhang, J. Pollock, J. Janjic, "Cyclooxugenase-2 Inhibiting Perfluoropoly (Ethylene glycol) Ether Theranostic Nanoemulsions-In vitro study", PLoS One, vol.8, no.2, e55802, 2013.

[94] U. Bazylinska, J. Kulbacka, G. Chodaczek, "Nanoemulsion Structural Design in Co-Encapsulation of Hybrid Multifunctional Agents: Influence of the Smart PLGA Polymers on the Nanosystem-Enhanced Delivery and Electro-Photodynamic Treatment", Pharmaceutics, vol.11, no.405, pp.1-15, 2019.

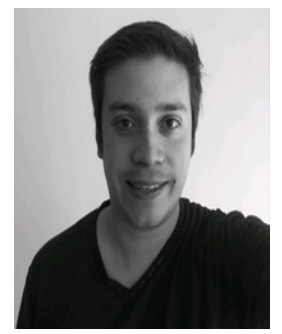

Felipe Andrés Silva Jerez. Ingeniero Civil en Biotecnología (Universidad San Sebastián, Chile. 2010-2017). Candidato a Máster en Innovación en Biociencias y BioIngeniería (Universidad San Sebastián, Chile. 2018-2021). Actualmente realiza su proyecto de tesis "Nanoemulsión con propiedades biomédicas a base de los compuestos bioactivos curcumina y micosporina para aplicaciones en el ámbito cosmético y del cuidado de la piel". Sus intereses se centran en el desarrollo de nanoemulsiones y sistemas coloidales.

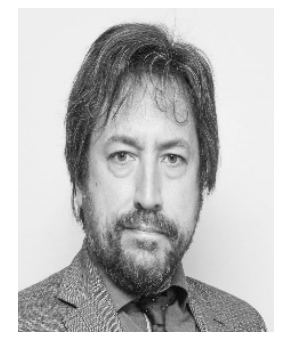

Patricio Alejandro Oyarzún Cayo. Ph.D en Biotecnología (University of Queensland, Australia. 2009-2012). Máster en Ciencias de la Ingeniería con Mención en Ingeniería Bioquímica (Universidad Católica de Valparaíso, Chile. 1998-2001). Bioquímico (Universidad Católica de Valparaíso, Chile. 1990-1996). Director de Investigación de la Universidad San Sebastián sede Tres Pascualas, Concepción (Chile). Sus intereses se centran en los ámbitos de la Inmunoinformática y Nanobiotecnología. 